GRUNDLAGEN DER MAGNETISCHEN

SIGNALSPEICHERUNG 


\section{ELEKTRONISCHES RECHNEN UND REGELN}

\section{Herausgegeben von}

Prof. Dr. HANS FRUHAUF - Prof. Dr. WILHELM KÄMMERER

Prof. Dr. KURT SCHRÖDER - Prof. Dr. HELMUT WINKLER

Sonderband 7

\section{GRUNDLAGEN DER MAGNETISCHEN SIGNALSPEICHERUNG}

I

\section{bearbeitet}

von

HORST VÖLZ

A K A D E M I E - V E R L A G - B E R L I.N 1968 


\title{
GRUNDLAGEN DER MAGNETISCHEN SIGNALSPEICHERUNG
}

\author{
BAND I \\ GRUNDLAGEN UND MAGNETKÖPFE
}

VON

CHRISTIAN KORISTKA, ZOLTAN VAJDA, HORST VÖLZ

Mit 136 Abbildungen und 17 Tabellen

A K A D E M I E - V E R L A G - B E R L I N 1968 
Erschienen im Akademie-Verlag GmbH, 108 Berlin, Leipziger Straße 3-4

Copyright 1968 by Akademie-Verlag Berlin

Lizenznummer : $202 \cdot 100 / 437 / 68$

Gesamtherstellung: VEB Druckerei „Thomas Müntzer", 582 Bad Langensalza



